\title{
Identification of Flexible Structures for Robust Control
}

\author{
Gary J. Balas and John C. Doyle
}

\begin{abstract}
This article documents our experience with modeling and identification of an experimental flexible structure for the purpose of control design, with the primary aim being to motivate some important research directions in this area. Initially, a multi-input/multi-output model of the structure is generated using the finite element method. This model is inadequate for control design, due to its large variation from the experimental data. Next, Chebyshev polynomials are employed to fit the data with single-input/multli-output (SIMO) transfer function models. Combining these SIMO models leads to a multi-input/multi-output (MIMO) model with more modes than the original finite element model. To find a physically motivated model, an ad hoc model reduction technique which uses a priori knowledge of the structure is developed. The ad hoc approach is compared with balanced realization model reduction to determine its benefits. Descriptions of the errors between the model and experimental data are formulated for robust control design. Plots of select transfer function models and experimental data are included.
\end{abstract}

\section{Introduction}

This article documents our experience with modeling and identification of a flexible structure for the purpose of control design. A sequence of techniques is presented for generating accurate nominal and uncertainty models of the experimental structure in a framework compatable with robust control design methods, specifically structured singular value $(\mu)$ methods $[1,2]$. There are

Presented at the 1989 American Control Conference, Pittsburgh, PA, June 21-23, 1989. Gary J. Balas is with the Department of Aerospace Engineering and Mechanics, University of Minnesota, Minneapolis, MN 55454. John C. Doyle is with the Department of Electrical Engineering, California Institute of Technology, Pasadena, CA 91125. This work was supported by a NASA Graduate Student Fellowship and by the Caltech President's Fund. no new theoretical results in the article, only the application of curve fitting and model reduction techniques. These techniques provide an ad hoc approach to system identification, though a more systematic method is desired. The primary aim of this article is to motivate some important research directions in this area.

Initially, a theoretical model of the flexible structure is developed using the finite element method. The natural frequencies and mode shapes of the model vary considerably from the experimental data. These variations (uncertainties) are attributed to inaccuracies in the mathematical model and the interaction between the actuators and the structure. Chebyshev polynomial curve fitting is used to formulate single-input/multi-output (SIMO) transfer function models from experimental data, to provide more accurate models. A multi-input/multi-output (MIMO) transfer function model is constructed from the individual Chebyshev SIMO models, with the resulting $\mathrm{MIMO}$ model having the same number of states as the sum of the states of each SIMO model. This leads to a number of excess states in the MIMO model which are not motivated from the physics of the problem. A direct MIMO curve fitting method has been developed [3], but its implementation presents some numerical problems, and it is not used.

Two methods are used to construct a reduced order MIMO model from the SIMO models: one uses physically motivated arguments to combine states of the system, and the other balanced truncation. These methods provide varying degrees of accuracy in approximating the experimental data. Quantitative descriptions of the variations between the experimental data and the mathematical models need to be developed for control design. These descriptions determine the tradeoff between achievable performance and robustness of the control design. A controller synthesized for a physical system not within the set of plants described by the nominal and uncertainty model may be unstable or exhibit poor performance when implemented on the physical system. However, if the uncertainty descriptions are overly conservative, system models may be included inthe set that severly limit the performance of the closed loop system. Therefore, tight uncertainty bounds are required to synthesize control designs that achieve high performance onthe "real" system [4].

It is apparent that there are a number of shortcomings associated with these methods for modeling and identification of flexible structures for control design. Some of the issues in need of attention are:

1) More straightforward and direct MIMO modeling.

2) Identification methods that produce nominal models with both perturbations and noise. For control design, there is an explicit need for uncertainty models to account for model inaccuracies.

3) Improved mathematical models. Of particular importance is the problem of producing nominal and uncertain models using the finite element method. Such models are likely to be conservative, but will be better than those used herein in the initial control design.

4) Integration of the identified model with the finite element method. There should be a way of incorporating post-identification models of the system into updating the finite element model of the system. Progress in these areas will lead to a more integrated framework in which structural and control design for flexible structures can be better performed.

\section{Objective}

The objective of the Caltech flexible structure experiment is to examine active control techniques for vibration suppression of flexible structures. The performance requirement is to significantly attenuate vibration of the first six flexible modes, measured at the sensors, via active control. To achieve these tight performance specifications, an accurate transfer function model and uncertainty descriptions of the structure are required. 
The control design methodology is based on the $\mu$-framework. The nominal structural model, uncertainty descriptions and performance specifications are combined to form an interconnection structure $P$ used in the control problem formulation. Fig. 1 depicts the general interconnection structure used in the $\mu$-analysis and synthesis methodology, with $\Delta$ representing norm-bounded perturbations and $K$ the control design.

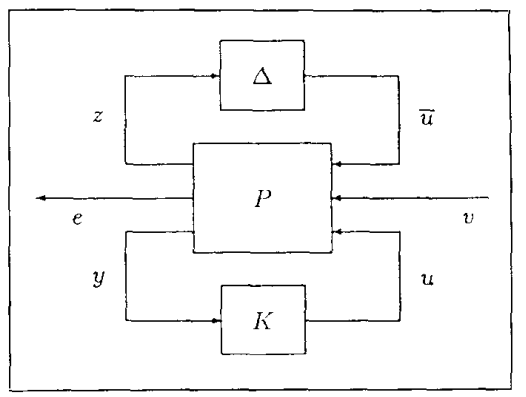

Fig. I: General interconnection structure.

The u-framework most naturally uses frequency domain descriptions of the plant model, uncertainties and performance specifications. Therefore, transfer function models are developed to accurately describe the experimentally derived Bode plot data in the frequency domain of interest. Once a baseline model is defined, its variation from the experimental data is quantified and used to develop ad hoc uncertainty models that bound these variations.

\section{Experimental Flexible Structure}

The Caltech experimental flexible structure is designed to include a number of attributes associated with large flexible space structures $[2,5]$. These include lightly damped, closely spaced modes, colocated and non-colocated sensors and actuators, and numerous modes in the controller crossover region. In addition to these considerations, expandability of the structure is a desired feature. Modular construction provides a means for increasing the modal density in a frequency range of interest.

The initial experimental structure, Fig. 2. consists of two stories, three longerons (columns) and three noncolocated sensors and actuators. The first story columns are $0.838 \mathrm{~m}$ (33 in) long, with $6.35 \mathrm{~mm}(1 / 4$ in) diameter aluminum rods. The second story columns measure $0.759 \mathrm{~m}$ (29.9 in) with $4.76 \mathrm{~mm}$ (3/16 in) diameter aluminum rods.
Including the platforms, the height of the structure is $1.651 \mathrm{~m}$ (65 in). The two platforms are in the shape of equilateral triangles with a $0.457 \mathrm{~m}$ (18 in) base. The longerons are connected between the stories via a triangular mating fixture and three bolts. This allows for the easy addition of stories to the structure. All the longerons are shrunk fit and welded to their mating brackets, to reduce the effects of joint nonlinearities.

The first story platform is a $9.52 \mathrm{~mm}(3 / 8$ in) thick plate of aluminum, weighing 2.36 $\mathrm{kg}(5.2 \mathrm{lb})$, with diagonal mounting brackets for attachment of the actuator diagonals. The second story platform is a $6.35 \mathrm{~mm}(1 / 4 \mathrm{in})$ thick plate of aluminum with mounting holes for three accelerometers. It weighs $1.55 \mathrm{~kg}$ $(3.4 \mathrm{lb})$. A small offset mass is located on the second story platform to lower the torsional natural frequencies. The entire structure hangs from a mounting structure fixed to the ceiling. This alleviates the problem of buckling of the longerons. The three actuators are attached to the mounting structure, and act along the diagonals of the first story. The three sensors, accelerometers. are located on the second bay platform.

The two stories are designed to have the same first bending natural frequency. This is obtained by selecting the stiffness of the second story columns to be $1 / 4$ the stiffness of the first. with a similar ratio between the masses of the two stories. The ratio of stiffness and masses allows the interaction of the two stories to decrease the first bending

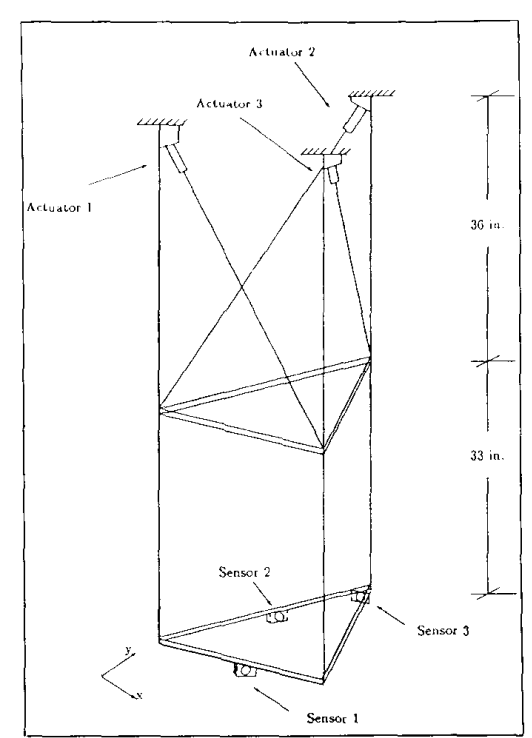

Fig. 2: Phase I Caltech flexible structure. natural frequency of the combined structure, without significantly spreading out the remaining modes. Interaction of the stories leads to poor performance of a colocated velocity feedback at the voice coil actuators.

\section{Voice Coil Actuator}

The actuators are a voice coil type design. built by Northern Magnetics Inc., that produce a force proportional to an input voltage. The actuators are rated at $\pm 1.37 \mathrm{~kg}$ (3 lb) of force at $\pm 5 \mathrm{~V}$, and have a bandwidth of $60 \mathrm{~Hz}$.

\section{Accelerometers}

The sensors are Sunstrand QA-1400 accelerometers. These are mounted on the second (lower) story platform, along the $x$ axis, $y$-axis, and at $45^{\circ}$ to both axes. The accelerometers are extremely sensitive and have a flat frequency response between 0 and $200 \mathrm{~Hz}$. The noise associated with them is rated at $0.05 \%$ of the output at $0-10 \mathrm{~Hz}$ and $2 \%$ at $10-100 \mathrm{~Hz}$. The sensors are scaled for accelerations of approximately $0.016 \mathrm{~g} / \mathrm{V}$. This provides a maximum $\pm 5 \mathrm{~V}$ output at peak accelerations of the input disturbance. The accelerometer output is conditioned by a $100-\mathrm{Hz}$ fourth-order Butterworth filter before input into the $\mathrm{A} / \mathrm{D}$; this provides attenuation of the high frequency signals and noise.

\section{Modeling}

A model of the structure which relates input signals to system outputs is needed for control design purposes. Initially, an input/output model is derived using the finite element method. In addition to this model, a transfer function model between the actuators and sensors is determined, by the ad hoc technique presented in this article. State space models of these are then constructed.

The finite element model (FEM) of the experiment provides a first approximation to the natural frequencies and mode shapes of the structure [6]. The columns and diagonals are treated as space frame elements having three translational and three rotational degrees of freedom at each node, and a torsional stiffness and bending stiffness in two directions. The longerons and diagonals are circular bars which have the same bending stiffnesses in two directions. Both are modeled as having fixed-fixed ends due to the welding of their end connections. The accelerometers, mounting brackets, platforms and additional weights on the structure are 
modeled as lumped masses. The inertia properties of each are taken into account in the finite element description.

The diagonals in the first story ride on the bearings of the voice coil actuators when the control system is not activated. No force is exerted in the open-loop configuration. The voice coil actuators are modeled as having free axial motion and being fixed in the two transverse directions. In reality, the diagonals ride on bearings that exhibit some stiction, friction, and free play. The bearings cause the damping levels to vary with the excitation amplitude. The degrees of freedom associated with vertical motion (along the longerons) are neglected in the analysis, since they correspond to high frequency modes outside the bandwidth of the current control design objectives.

The first six global modes are of interest for control purposes. The first group of local modes, involving bending of the longerons, occurs in the frequency range of 37 to $43 \mathrm{~Hz}$. The local modes are accounted for by uncertainty descriptions in the control design to insure that they are not destabilized. Attenuation of their vibration is not a performance criteria. Table I contains a list of natural frequencies derived from the Nastran finite element model and experimentally derived natural frequencies and damping levels. The variation in the natural frequencies is believed to be caused by two phenomena: the wires to the accelerometers were tightly fixed to the columns and pulled taut, thereby adding stiffness to the longerons; and stiction/friction associated with the voice coil actuators resulted in amplitude-dependent damping levels.

\section{Real Time Control Implementation}

A 5400 Masscomp computer is used for real time control. The controllers consist of 3-input/3-output systems that are implemented at $200 \mathrm{~Hz}$. The processor is capable of implementing a 60th-order control law, in modal coordinates, at this sample rate.

\section{Experimental Transfer Functions}

A more accurate input/output description of the structure can be derived experimentally. A white noise random process is used as an input signal to each voice coil actuator, with the accelerations due to this signal measured by the sensors. The accelerations are scaled to achieve a large signal to noise ratio for the disturbance excitation. With this in mind, the random noise signals are scaled accordingly. These scalings lead to a

\begin{tabular}{|c|c|c|c|c|}
\hline \multicolumn{5}{|c|}{ Table I } \\
Momping Ratios and Natural Frequencies of the Phase I Experiment \\
\hline \hline \multirow{2}{*}{ Mode } & $\begin{array}{c}\text { NASTRAN } \\
\text { Natural } \\
\text { Frequency }(\mathrm{Hz})\end{array}$ & $\begin{array}{c}\text { Experimental } \\
\text { Natural } \\
\text { Frequency }(\mathrm{Hz})\end{array}$ & Damping Ratio & Mode Type \\
\hline 1 & 0.991 & 1.17 & $1.8 \%$ & 1st bending \\
\hline 2 & 0.992 & 1.19 & $1.8 \%$ & 1st bending \\
\hline 3 & 2.004 & 2.26 & $1.0 \%$ & 1st torsional \\
\hline 4 & 2.069 & 2.66 & $1.6 \%$ & 2nd bending \\
\hline 5 & 2.100 & 2.75 & $1.8 \%$ & 2nd bending \\
\hline 6 & 3.832 & 4.43 & $0.9 \%$ & 2nd torsional \\
\hline
\end{tabular}

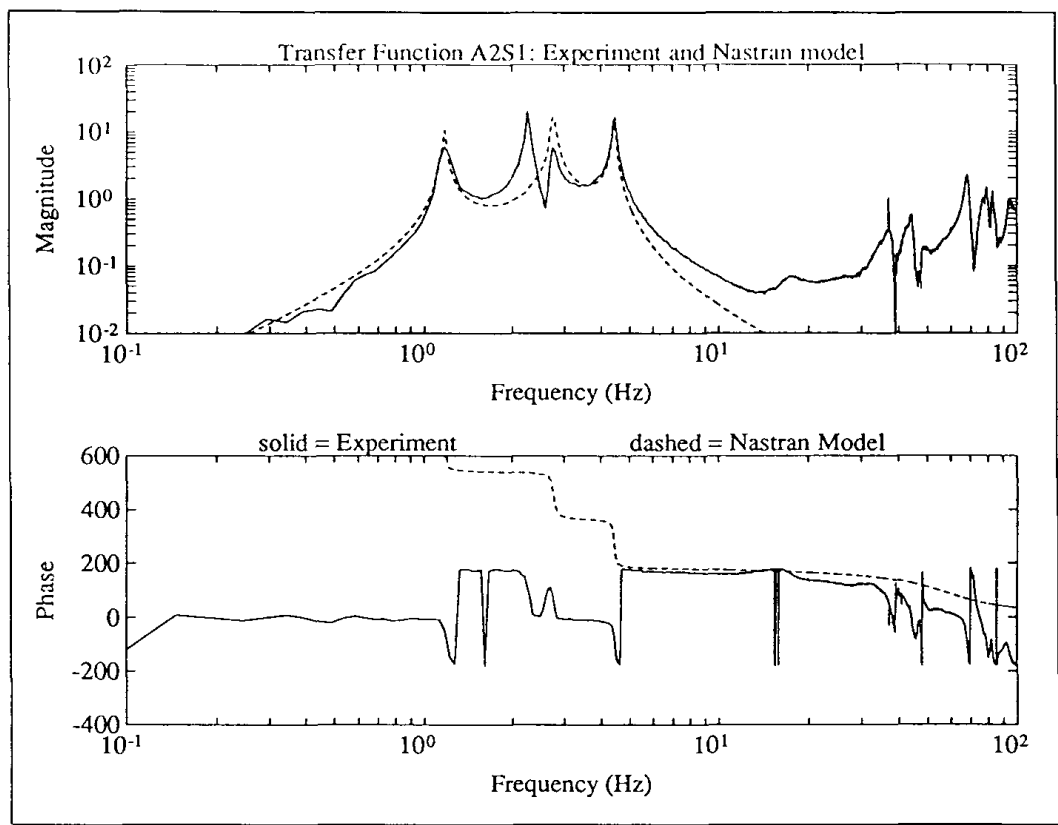

Fig. 3: Transfer function A2S1: Experiment and Nastran model.

maximum force output from the actuators of $\pm 0.5 \mathrm{lb}$ for the noise input. The input signals, in the form of voltage levels, are suppled by the Masscomp computer D/A converters to each voice coil actuator. The corresponding signals from the sensors are filtered by a $100-\mathrm{Hz}$ fourth-order Butterworth filter, and input to the Masscomp computer via the $A / D$ converters. A sample rate of $200 \mathrm{~Hz}$ is used for the identification experiments, the same as used in the closed-loop control experiments. Each single-input to multi-output identification experiment is run for a total of
409.6 s (81 920 sample points).

A Fourier transform of the time history is performed on each input/output pair. For the Fourier transform, the data is chopped into windows of length 4096 data points. Each window overlaps the previous one by 2048 data points. A total of 39 windows of data is averaged for each transfer function. Hamming windowing is used on the time domain data, to improve the smoothing properties of the frequency spectrum. A total of nine transfer functions are deterinined. The Bode plots of two experimentally derived transfer 


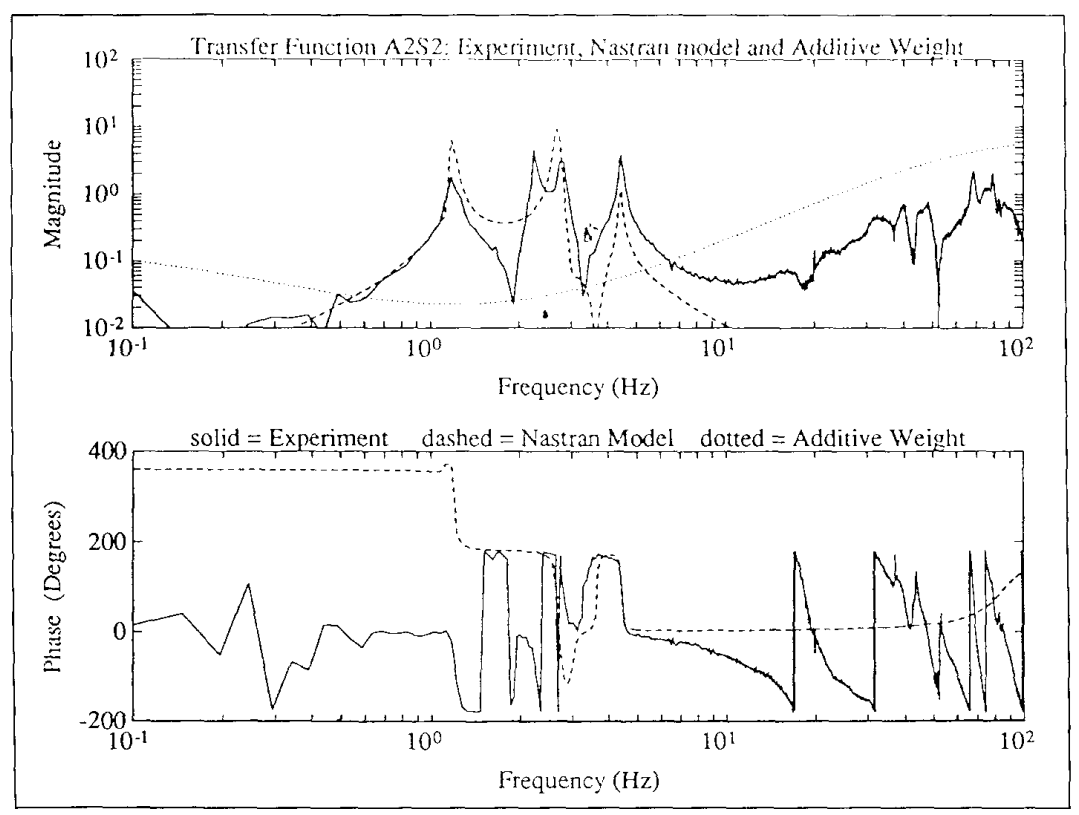

Fig. 4: Transfer function A2S2. Experiment. Nastran model and additive weight.

functions are shown in Figs. 3 and 4 . together with the Bode plots of the finite element transfer function models. In the plots of the FEM transfer functions. we have substituted the experimentally derived natural frequencies and damping ratios of the structure for the Nastran ones. The acronym $\mathrm{A} i \mathrm{~S} j$ in the figure legends denotes a transfer function from actuator $i$ to sensor $j$.

Notice that the agreement is poor between the theoretical finite element model and the experimental data in the frequency range of interest, 0.5 to $5.5 \mathrm{~Hz}(3.14$ to $34.5 \mathrm{rad} / \mathrm{s})$. The FEM had unacceptable variations from the experimental data. Therefore, new models are developed from the experimental data to more accurately represent the input/output behavior of the real system. The improved transfer functions model is used to design active control systems for vibration suppression.

\section{Uncertainty Descriptions}

The $\mu$-framework requires the nominal structural transfer function model to be formulated as a linear time invariant (LTI) system. Though this model might describe the physical system accurately, any model is only an approximation of the physical system. There is always some "uncertainty" present, even when the underlying process is essentially linear. This can be due to not knowing the physical parameters exactly. neglecting the high frequency dynamies, or making invalid assumptions in formulating the model. These inaccuracies can be described in numerous ways. such as: bounds on the parameters of a linear model. bounds on the nonlinearities. and frequency domain bounds on transfer function models. One needs to account for the variation between the mathematical model and the "real" system in the control design.

We will restrict our attention to frequency control design model. This leads to the flexible structure being described by a nominal LTI system and frequency varying uncertainty models in the control problem formulation. These models allow us to account for the variation in experimental data at specific frequency points. For example, a frequency response experiment is performed to establish upper and lower bounds on both the magnitude and phase of the real system as a function of frequency. Variations in the data are then approximated by disk shaped regions in the complex plane. which lead to either a multiplicative or additive uncertainty plant model. together with the uncertainty models. is used to define a set of plants within which the "real" physical system is assumed to lie. $\mu$-analysis techniques measure the stability and performance of control laws for these prescribed models. while the 1 synthesis methodology is employed to optimize the control laws to meet the stability and performance requirements. domain descriptions of uncertainty for the description of the bounds [7]. The nominal
The plant transfer function can be described by $P(s)+\Delta P(s)$. where $P(s)$ is the nominal plant model and $\Delta P(s)$ is an unknown perturbation. Every transfer function is taken to be a function of $s$, therefore the (s) notation will be dropped henceforth.

Consider a SISO system with $\Delta P$ bounded across frequency by a weighting function $W_{4}$ which is a real-rational, stable minimum phase transfer function and $\delta$, a norm bounded complex number $\delta \mid \leq 1$ such that

$$
|\Delta P(j \omega)<| W_{u}(j \omega) \delta \mid
$$

for all

$$
0 \leq \omega \leq \infty . \quad|\delta| \leq 1 . \quad s=j \omega .
$$

$\omega$ represents individual frequency points. The set of plants described by this uncertainty is given by

$\hat{P}(j \omega)=P(j \omega)+W_{d t}(j \omega) \delta|\Delta| \leq 1$.

Equation (2) is referred to as an additive uncertainty description and (1) defines the bound on the allowable additive uncertainty. $\delta$ is an unknown complex constant at each frequency. This assumption is implicit in the formulation of the robust control analysis and synthesis methods. For a multivariable system. the magnitude bounded scalar uncertainty $\delta$ can be replaced with $\Delta$, a norm bounded complex matrix such that $\bar{\sigma}(\Delta) \leq 1$. The magnitude bounds in (1) would be replaced by norm bounds on the maximum singular value.

Additive uncertainty is used to account for unmodeled dynamics in flexible structures. These unmodeled dynamics are a result of low or high frequency modes outside the desired control bandwidth. They are not modeled because the mode representations are inaccurate. the frequencies and mode shapes associated with them vary, or because low order design models are desired. Unmodeled modes need to be accounted for in the problem formulation. so as not to destabilize them with the control design. The size of the additive uncertainty weight is selected to encompass the transfer function response of these modes at each frequency.

Another approach to modeling errors involves multiplicative uncertainty descriptions. Consider a SISO transfer function initially. Defining $W_{n}(j \omega)=W_{a}(j \omega) / P(j \omega)$, we can describe a set of plants by

$$
\hat{P}(j \omega)=P(j \omega)\left(1+W_{m}(j \omega)\right)
$$

and

$$
\frac{\left|W_{u}(j \omega)\right|}{P(j \omega)}=\left|W_{n}(j \omega)\right| .
$$


Equation (3) is referred to as a multiplicative uncertainty description, and states a bound on the allowed multiplicative uncertainty.

Multiplicative uncertainty descriptions are used to account for relative variations in input or output signals and, in multivariable systems, to account for directionality of signals. Additive and multiplicative uncertainty descriptions are formulated for control design based on engineering judgement, since no systematic approach, in conjunction with system identification, is available for their selection. Other techniques to determine these uncertainty descriptions have been proposed, but are not addressed in this article $[8,9]$.

\section{Chebyshev Polynomial Curve Fitting}

Chebyshev polynomials have previously been used in FFT signal analyzers to curve fit measured transfer function data of singleinput/single-output (SISO) systems [10]. This technique was extended to single-input/multiple-output (SIMO) systems and has been applied successfully to experimental data [3]. The same technique is employed to develop SIMO transfer function models for the Caltech flexible structure experiment.

The transfer function equation, which is nonlinear in the coefficients, is transformed into a linear equation by multiplying through by the denominator $g(s) d(s)-n(s)=0$, where $s$ is defined as $j(\omega)$

$$
\begin{aligned}
g(s) & =\frac{n(s)}{d(s)} \\
& =\frac{n_{0}+n_{1} s+n_{2} s^{2}+\cdots+n_{N} s^{N}}{d_{0}+d_{1} s+d_{2} s^{2}+\cdots+d_{N} s^{N}} .
\end{aligned}
$$

The transfer function data is a set of complex numbers $g(j \omega)$ at various frequency points $\omega$. Separating this equation into real and imaginary parts, two real equations are produced for each value of $\omega$. Written in matrix form, they form a linear least squares problem, inf $f_{|,|=1}\|\hat{A} x\|$. The real vector $x$ contains the polynomial coefficients of $n(s)$ and $d(s)$.

A problem with this approach is that the matrix $\hat{A}$ is ill-conditioned. This is due to the ratio $n(s) / d(s)$ being very sensitive to small changes in their coefficients. To alleviate this problem, the numerator and denominator are written as sums of Chebyshev polynomials and, therefore, indirectly as sums of powers of $s$.

At each frequency point $\omega$, the equation $g(j \omega) d(j \omega)-n(j \omega)$ contributes two rows to the matrix $\hat{A}$. A weighting can be associated with each individual frequency point, allowing the accuracy of the fit to be traded off for different frequency ranges. Each row is normalized by $|g(j \omega) d(j \omega)|$, using an estimated $d(j \omega)$ to achieve a constant relative accuracy (in log magnitude and phase) at each frequency.

The algorithm used to fit the data with Chebyshev polynomials is as follows [3] :

1) Read in data points $g(j \omega)$ and associated weights.

2) Construct $A=\hat{A}^{T} \hat{A}$

3) Solve for $x$ to minimize $x^{7} A x$.

4) Use $x$ to build $d(j \omega), n(j \omega)$.

5) Using $|g(j \omega) d(j \omega)|^{-1}$ as a weight, cycle back to Step 2.

6) When the process converges, compute the state space realization of $n(s) / d(s)$.

For the SIMO case, the denominator has the same dynamics as in the SISO case. Therefore, by extending the number of numerator coefficients $n(s)$ one can address the SIMO case in a fashion similar to the SISO case.

One problem with the Chebyshev curve fitting method is that it does not guarantee that a stable transfer function will be fit to the raw data. However, given that the frequency domain data reflects a stable system, and the polynomial approximation is a good fit to the data, the stability properties of the two are usually comparable. This was not an issue in this instance. Once the singleinput/multi-output transfer functions are fit with Chebyshev polynomials, the models are converted to a state space description.

\section{MIMO Transfer Function Model}

A multivariable transfer function model is constructed from the individual SIMO models. This model has the same number of states as the sum of each SIMO model, leading to an excess number of states in the MIMO model which are not physically motivated. A singular value decomposition (SVD) model reduction technique, based on an a priori model of the system, is developed to produce a MIMO transfer function model of the same order as the finite element model. The Chebyshev polynomial curve fitting and SVD-based model reduction techniques are used in sequence to form a system identification method for flexible structures.

Based on the finite element model and physical data, the experimental structure has only six natural frequencies and mode shapes between 0.5 and $5.5 \mathrm{~Hz}$. This is the frequen- cy range in which an accurate multivariable model of the structure is required for control design. Therefore, the 3-input/3-output multivariable transfer function model of the structure should have six modes associated with it. The SIMO Chebyshev curve fitting technique is used to develop transfer function models from each actuator to the three accelerometer sensors. These models contribute four modes to the total system model for each input. Although all six modes are excited by each actuator, only four modes appear distinctly in the experimental data. After fitting the individual SIMO transfer functions, twelve modes comprise the 3 input/3-output transfer function model. One would like to take advantage of the physical knowledge of the problem to reduce the twelve-mode model to a six-mode model.

\section{Ad Hoc Model Reduction Technique}

This model reduction technique requires an a priori knowledge of the flexible structure experiment. Modes in the SIMO models are grouped together based on their natural frequencies and the theoretical model. Four groups of modes are defined in the frequency range of interest. These groups include the first bending modes, first torsional mode, second bending modes and the second torsional mode. SVD is used to reduce the modes present in the Chebyshev MIMO model, to the number of physically motivated modes.

The experimental structure has two first bending and second bending modes present in the frequency range of interest. The two modes associated with the first bending mode have approximately the same natural frequency, as do the second bending modes. The bending modes have similar natural frequencies, but their mode shapes are perpendicular to one another. In the individual transfer functions, it is hard to differentiate between the individual bending modes with similar natural frequencies. Hence in fitting the Chebyshev polynomial models to the experimental data, the first and second bending modes are treated as having only one mode each. Each SIMO model consists of one first bending mode, a first torsional mode, a second bending mode and a second torsional mode.

Combining the SIMO Chebyshev polynomial transfer function models for actuators 1 and 2, a 2-input/3-output eight-mode model is formed. Because there are two first and second bending modes, the coefficients associated with both the first and second bending modes remain in the model. This model contains two modes that are not physically motivated. It is found that each 
torsional mode in the eight-mode model has two nearly identical natural frequencies associated with it, accounting for the two extra modes. The extra modes are due to the torsional response showing up predominantly in both sets of transfer functions from actuators 1 and 2 to the three sensors. From the physics of the problem, there is only one mode associated with each torsional natural frequency. A common, one-mode model for each torsional mode must be unraveled from the two SIMO transfer function models. To see how this might be done, a modal description of the experimental structure is constructed.

The voice coil actuators input a force to the structure, and accelerations are measured. Assuming modal damping, a SISO transfer function model relating force input to acceleration output can be developed for individual modes. For the $i$ th mode, it has the form:

$$
\frac{b_{i} c_{i} s^{2}}{s^{2}+2 \zeta_{i} \omega_{i} s+\omega_{i}^{2}}
$$

Rewriting the transfer function in strictly proper form yields

$$
b_{i} c_{i}-\frac{2 s \zeta_{i} \omega_{i} b_{i} c_{i}+\omega_{i}^{2} b_{i} c_{i}}{s^{2}+2 \zeta_{i} \omega_{i} s+\omega_{i}^{2}} .
$$

The coefficients $b_{i} c_{i} . \zeta_{i}$ and $\omega_{i}$ are determined from the Chebyshev polynomial models. Notice that only the combined scalar $b_{i} c_{i}$, can be determined uniquely. Unfortunately, this does not allow for the identification of the individual modal coefficients $b$ and $c_{;}$associated with each mode. However. the identified coefficients are within a scalar transformation of the modal coefficients.

The transfer functions, written in state space form, are described by

$$
G(s)=\left|\begin{array}{c|c}
A & B \\
\hline C & D
\end{array}\right| \doteq D+C(s I-A)^{-1} B .
$$

The $D$ term derived from curve fitting is often inaccurate, hence disregarded since it is outside the frequency range in which the data is fit.

One way to determine each component from the curve fitting data is to replace $s$ by $j \omega$, and evaluate the strictly proper transfer function associated with each individual mode at $\omega=0$. Consider an individual mode of an identified SISO transfer function $\tilde{G}(s)$ whose state space representation is of the form

$$
\begin{aligned}
\tilde{G}(s) & =\left[\begin{array}{cc|c}
0 & 1 & 0 \\
-\omega_{i}^{2} & -2 \zeta_{i} \omega_{i} & b_{i} \\
\hline-c_{i} \omega_{i}^{2} & -2 c_{i} \zeta_{i} \omega_{i} & d_{i}
\end{array}\right] \\
& =d_{i}-\frac{2 s \zeta_{i} \omega_{i} b_{i} c_{i}+\omega_{i}^{2} b_{i} c_{i}}{s^{2}+2 \zeta_{i} \omega_{i} s+\omega_{i}^{2}} .
\end{aligned}
$$

Evaluating this equation at $s=0$. with the

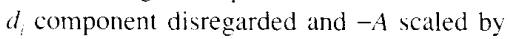
-1 . leads to

$$
C(A)^{-1} B=\frac{b_{i} c_{i} \omega_{i}^{2}}{\omega_{i}^{2}}=b_{i} c_{i}
$$

The individual model components $b_{i} ;$ can be determined for each mode with this method. The same idea can be applied to multiple input and output pairs with a single mode. Instead of a scalar, a full matrix would be determined.

A priori it is known that only one or two modes are present in the data at each modal natural frequency. In the case of the experimental truss structure. it is known that there are two first bending modes whose natural frequencies are close, one first torsional, two second bending modes with close natural frequencies, and one second torsional mode. Using this information. a SVD of the matrix is performed and the dominant mode is kept for the torsional case. while two are kept for the bending cases. For one mode, the maximum singular value and its associated right and left eigenvectors determine the $b_{\text {, }}$ and $c$ coefficients.

The singular value decomposition for an $n \times m$ matrix $A$ [1]\}, is given by the following, where $U$ and $V$ are unitary matrices with column vectors denoted by $U$

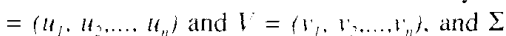
contains a diagonal nonnegative definite matrix of singular values arranged in descending order:

$$
A=U \Sigma V^{*}=\sum_{i=1}^{K^{\prime}} \sigma_{i}(A) u_{i} v_{i}^{*}
$$

The $C$ matrix, corresponding to the output direction of the mode. is constructed from the maximum singular value and the unitary $U$ matrix. For a single mode, the $C$ matrix is given by $C=\sigma_{1} u_{1}$, where $C$ is a vector of the length of the number of outputs. The $B$ matrix, constructed from the $V$ matrix, is given by $B^{T}=v$, which is the right singular vector associated with the maximum singula value. The matrix $B^{l} C$ has the corresponding $b_{i} c$; matrix elements associated with input $i$ and output $j$. For muttiple modes, the $B$ and $C$ vectors would be matrices of size number of modes $\times$ number of inputs) and (number of outputs $\times$ number of modes). respectively.
These matrices could be derived in a similar fashion.

This approach is used to transform the 3 input/3-output twelve-mode model developed from Chebyshev polynomials into a 3 -input/3-output six-mode model which agrees with the physical properties of the structure in the frequency range of interest. The three SIMO transfer function models have three modes describing the first bending modes. three modes for the first torsional mode, three modes describing the second bending modes, and three modes for the second torsional mode. Applying the SVD-based reduction method to these modal groups led to the development of a six-mode MIMO transfer function model of the experimental flexible structure with two first bending modes, a first torsional mode, two second bending modes and a second torsiona! mode.

Presented in Figs. 5 and 6 is a comparison among the Bode plots of the transfer functions from a) the experimental data, b) the SIMO Chebyshev polynomial model method. and c) the six-mode MIMO method derived using the techniques described above. The frequency range of interest for fitting of the Chebyshev polynomial model is between 0.5 and $5.5 \mathrm{~Hz}(3.14$ and $35 \mathrm{rad} / \mathrm{s})$.

\section{Bulanced Model Reduction}

The method of model reduction based on balancing is also applied to the twelve-mode MIMO model constructed from the three Chebyshev SIMO models. The objective is the same as before, to obtain a six-mode model from the Chebyshev MIMO twelvemode model. This method requires no physical knowledge of the system it is trying to approximate.

The balanced model reduction technique computes an $m$ th order reduced model $G_{n}$ of a possibly nonminimal $n$th order system $G$ such that certain conditions hold:

$$
\begin{gathered}
G_{m}=C_{m}\left(s I-A_{m}\right)^{-1} B_{m}+D_{m} \\
G=C(s I-A)^{-1} B+D \\
\left\|G(j \omega)-G_{m}(j \omega)\right\|_{\infty} \leq 2 \sum_{i=m+1}^{n} \sigma(i) .
\end{gathered}
$$

In this notation, $\sigma(i)$ are the square-roots of the eigenvalues of the controllability and observability grammians. These are also the Hankel singular values of $G(s)$ [12.13.14].

A six-mode MIMO model is developed using this technique. The reduced order transfer function model matches the original 


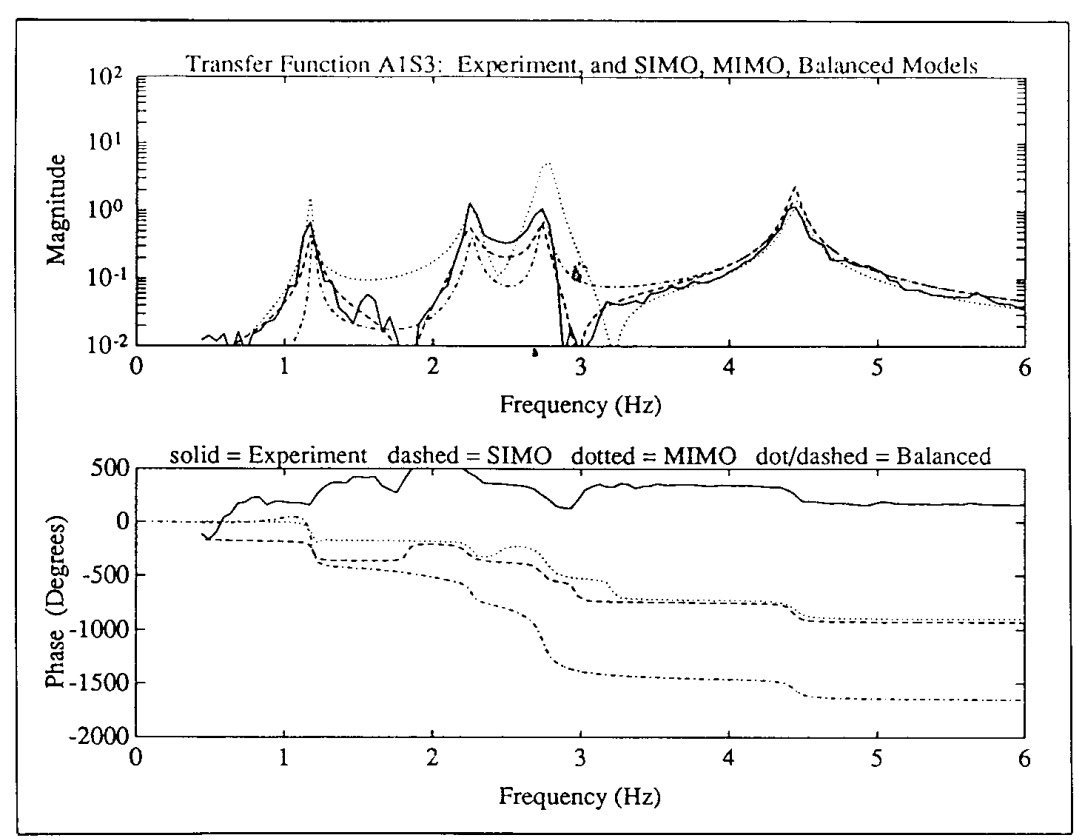

Fig. 5: Transfer function AIS3: Experiment and SIMO, MIMO, balanced models.
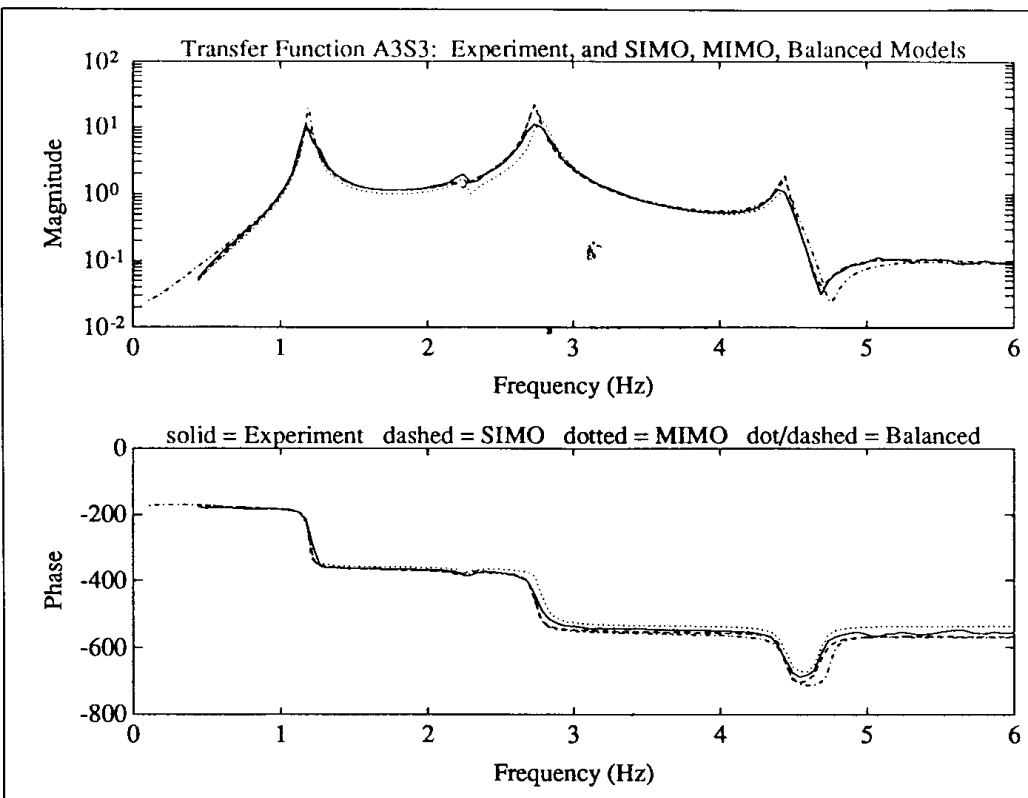

Fig. 6: Transfer function A3S3: Experiment and SIMO, MIMO, balanced models.

twleve-mode MIMO quite well. The corresponding natural frequencies and damping values also closely match those in the Chebyshev model. The corresponding Bode plots are shown in Figs. 5 and 6 , and are compared therein to those from the Chebyshev models and the experimental data.

\section{Selection of Uncertainty Weights}

Uncertainty models are developed to account for variation between experimental data and the MIMO model for control design. An additive uncertainty weight is used to describe the low frequency variation (below $0.5 \mathrm{~Hz}$ ) and the high frequency modes (above $5.5 \mathrm{~Hz}$ ) not included in the design model. The additive uncertainty transfer function weight, shown in Fig. 4, is given by

$$
\begin{aligned}
& W_{\text {additive }} \\
& \quad=8.0 \frac{(s+6)(s+12)(s+24)}{(s+0.6)(s+400)(s+400)} .
\end{aligned}
$$

This weighting requires use of the $\mu$ control design methodology to gain stabilize the high frequency modes not included in the design model, and to limit the controller gain at low frequency. Input and output multiplicative uncertainties are included, to model variations between the experimental data and the MIMO model in the frequency range of 0.5 to $5.5 \mathrm{~Hz}$. There is little variation in the uncertainty in this range. Therefore, both multiplicative uncertainties are taken to be scalars $[4,5]$. The input and uncertainty levels are selected to be $4 \%$.

\section{Experimental Data and Models}

Three different multivariable models are developed for the experimental data in the frequency range of interest. The first model, SIMO, is the Chebyshev SIMO transfer function model for each actuator input. A twelve-mode MIMO model is developed by combining the three Chebyshev SIMO models. The second model, MIMO, is the reduced, six-mode MIMO transfer function model that was formed using the ad hoc model reduction technique. The third model, "Balanced", is a six-mode MIMO model formed by applying balanced model reduction to the Chebyshev SIMO transfer function models.

As one would expect, the Chebyshev SIMO models provide the best fit to the experimental data. This is because the other two models only approximate the Chebyshev model. The poorest fit occurs in the Bode plot representing the transfer function between actuator 1 and sensor 3 . Since actuator 1 excites the direction perpendicular to sensor 3, the magnitude of the transfer function is an order of magnitude below that of the other actuator 1 transfer functions. The curve fitting technique applies a maximum magnitude error criteria to fit the data, which accounts for this discrepancy.

The ad hoc technique achieves a very good fit to all the experimental Bode plot data, except from actuator 1 to sensor 3 . The magnitude and phase characteristics of all the 
other Bode plots are well matched. The balanced model reduction also fits the data well, with the notable exception of the Bode plots associated with the transfer functions A2S2 and A1S3. The balanced model transfer function from actuator 2 to sensor 2 has problems with the interlacing of the poles and zeros associated with the second bending modes. Overall, the balanced model reduction method performed quite well. considering it required no knowledge of the dynamic characteristics of the system. The ad hoc technique produced the best correspondence between the six-mode model and the experimental data.

\section{Conclusions}

The finite element model of the structure provided a physical understanding of the dynamic characteristics of the first six modes. Unfortunately, this model had considerable error in the determination of the natural frequencies and mode shapes of the structure. To obtain a more accurate model of the experimental data, Chebyshev polynomials were employed to curve fit the data. This approach proved successful in deriving SIMO transfer function models for each actuator input, which accurately fit the data in the frequency range of 0.5 to $5.5 \mathrm{~Hz}$. To form a MIMO model, the three SIMO models were combined. A shortcoming of this combination was that additional modes were present in the resulting model, that were not physically motivated from the finite element analysis. The resulting Chebyshev MIMO model contained 12 modes, whereas the finite element model had only six.

The ad hoc model reduction technique provided the best fit to the experimental data for a six-mode model consistent with the finite element analysis. The balanced model reduction also provided a consistent model which fit the data. An a priori knowledge of the physical system aids in providing an accurate transfer function model corresponding to the physical data.

The problem with all three approaches is that they don't provide models which are readily useful for control design. A series of ad hoc assumptions and fixes are required to fit them into the robust control framework. The uncertainty descriptions developed to account for model error were determined from engineering judgment, rather than by a systematic approach. It is therefore difficult to verify their accuracy. As discussed in the introduction. there are a number of issues that need to be resolved in the area of identification for control design. Although the developed models proved very useful for our research, we do have a great interest in approaching system identification in a manner more consistent with the robust control framework.

\section{Acknowledgments}

The authors would like to thank Dr. Harold Stalford for his input and Dr. R. Lane Dailey for the Chebyshev polynomial curve fitting software.

\section{References}

111 J.C. Doyle. Lecture notes on advances in multivariable control, presented at ONR/Honeywell Workshop on Advances in Multivariable Control. Minneapolis. MN. Oc1. 1984.

[2] G.J. Balas and J.C. Doyle. "Robust control of flexible modes in the controller crossover region," in Proc. Amer. Control Conf.. Pittsburgh. PA. June 1989.

[3] R.L. Dailey and M.S. Lukich. "MIMO transfer function curve fitting using Chebyshev polynomials." presented at SIAM 35th Anniversary Meet.. Denver. CO. 1987.

[4] G.J. Balas and J.C. Doyle. "Robustness and performance tradeoffs in conlrol design for flexible structures." to be presented.

[5] G.J. Balas, "Robust control of tlexible structures: theory and experiments." Ph.D. dissertation. California Institute of Technology, Pasadena. CA. 1989

(6) O.C. Zienkiewicy. The Finite Element Method. Third Ed. London: McGraw-Hill, 1977.

17] J.C. Doyle. J. Wall and G. Stein. "Pertomance and robustness analysis for structured uncertainty." in Proc. 2/st LEEE Conf. Decision and Control. Dec, 1982.

[8/ M. Safonow and R. Chiang. "Model reduction for robust control: A Schur relative cror method." Int. J. Adaptive Conmol and Signal Processing. pp. 259-272. 1988

[9] M. Safonov, R. Chiang, and H. Flashner. "H. control synthesis for a large space structure," in Proc. Amer. Control Conf.. Atlanta. GA. June 1988.

[10] J.L. Adcock. "Curve fitter for pole-zero analysis." Hewlett.Packard I., Jan. 1987.

[11] R.A. Horn and C.A. Johnson. Matrix Analyis
Cambridge Press. 1985.

$112 \mid$ K. Glover. "All oprimal Hankel norm approximations of linear multivariable systems and their L. error bounds." Intl. I. Control. Vol. 39. No. 6. pp. 1115-119.3. 1984.

$113 \mid$ B.C. Moore, "Principal components analysis in linear systems: controllability, observability and model reduction," IEEE Trans Automatic Control. Vol. AC-26. pp. 17-31. Feb. 1981.

[1+] D. Enns, "Model reduction for control system design." Ph.D. dissertation. Stanford University. Stanford. CA. 1984

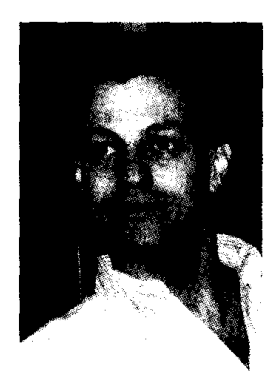

Gary J. Balas received the B.S. and M.S. degrees in civil and electrical engineering from the University of California. Irvine, and the Ph.D. degree in aeronautics from the California Institute of Technology in 1989. He is presently an Assistant - Professor in Aerospace Engineering and Mechanics at the University of Minnesola. Dr. Balas is co-organizer of the Theory and Application of Robust Control Short Course. and has consulted for a number of aerospace companies. His current rescarch interests include system identification and robust control with application to flexible structures, high performance flight vehicles, transportation, and robotics.

John C. Dovle received the B.S. and M.S. degrees in clectrical engineering from M.I.T. in 1977, and the Ph.D. degree in mathematics from the University of California-Berkeley in 1984. He has been a consultant to Honeywell Systems and Research Center since 1976. and an Associate Professor of Electrical Engineering at Caltech since 1986. His theoretical research interests include modeling and control of uncertain and nonlinear systems. matrix perturbation problems, and operator theoretic methods. Application interests include flexible structures, chemical process control, flight control, and control of unsteady fluid flow. Additional interests include gender issues in science and society and impact of control on system design. Dr. Doyle is the recipient of the Hickemell Award, the Eckman Award, the IEEF Control Systems Society Centennial Outstanding Young Engineer Award, and the Bernard Friedman Award. He is an NSF Presidential Young Investigator and an ONR Young Investigator. 